\title{
Ostéotomies Segmentaires en Implantologie et en Chirurgie Orthognathique
}

\section{B. Philippe (Paris)}

Les ostéotomies segmentaires qui consistent à mobiliser un fragment de la crête alvéolaire sont indiquées en implantologie pour restaurer un volume osseux insuffisant et/ou assurer un positionnement tridimensionnel satisfaisant de la crête alvéolaire. Si leur exécution avant l'insertion des implants relève du bon sens, elles peuvent aussi être réalisées après la pose des implants pour supprimer une fausse gencive inesthétique et/ou recouvrer un positionnement et une orientation physiologique des implants. En chirurgie orthognathique, les ostéotomies segmentaires permettent de corriger certaines anomalies de l'occlusion et de l'os alvéolaire en complément ou en remplacement de l'orthodontie. L'objectif de cette communication consiste à partir d'une série de 54 ostéotomies réparties chez 44 patients à présenter les indications, les principaux éléments de la technique opératoire et les principales complications peropératoires et post-opératoires toujours possibles de ces procédures chirurgicales mal connues. 